EPJ Web of Conferences 41, 06003 (2013)

DOI: $10.1051 /$ epjconf/20134106003

(C) Owned by the authors, published by EDP Sciences, 2013

\title{
Ultrafast vibrational dynamics of water confined in phospholipid reverse micelles
}

\author{
R. Costard ${ }^{1}$, C. Greve ${ }^{1}$, N. E. Levinger ${ }^{2}$, E. T. J. Nibbering ${ }^{1}$, T. Elsaesser ${ }^{1}$ \\ ${ }^{1}$ Max-Born-Insitut für Nichtlineare Optik und Kurzzeitspektroskopie, Berlin, Germany \\ ${ }^{2}$ Department of Chemistry, Colorado State University, Fort Collins, Colorado, USA
}

\begin{abstract}
We study the ultrafast dynamics of $\mathrm{OH}$ stretching and bending vibrations of water inside dioleoylphosphatidylcholine (DOPC) reverse micelles in a wide range of hydration. A strong hydration level dependence for the spectral diffusion rates is found and explained by the distinctly different environment for single water molecules bound to the anionic phosphate group. We show that the energy relaxation pathway of the $\mathrm{OH}$ stretching vibration at low hydration level involves the $\mathrm{OH}$ bending.
\end{abstract}

Phospholipids, building blocks of biological membranes, typically self-assemble into bilayers in aqueous solution. The amphiphilic nature of phospholipids allows the formation of a variety of other structures, including reverse micelles. We recently introduced reverse micelles composed of dioleoylphosphatidylcholine (DOPC), a phospholipid with hydrophobic tails and a hydrophilic head consisting of a phosphate $\left(\mathrm{PO}_{4}^{-}\right)$unit and a covalently linked choline group (cf. Figure 1). Such systems form when DOPC is dissolved in nonpolar solvents resulting in hydrophilic head groups pointing to the interior. Addition of water leads to hydration of the head groups as well as the buildup of nanoscopic water pools inside the reverse micelles (the water content is characterized by the parameter $\left.w_{0}=\left[\mathrm{H}_{2} \mathrm{O}\right] /[\mathrm{DOPC}]\right)$. Therefore, reverse micelles allow for detailed studies of the fundamental hydration interactions of phospholipids.
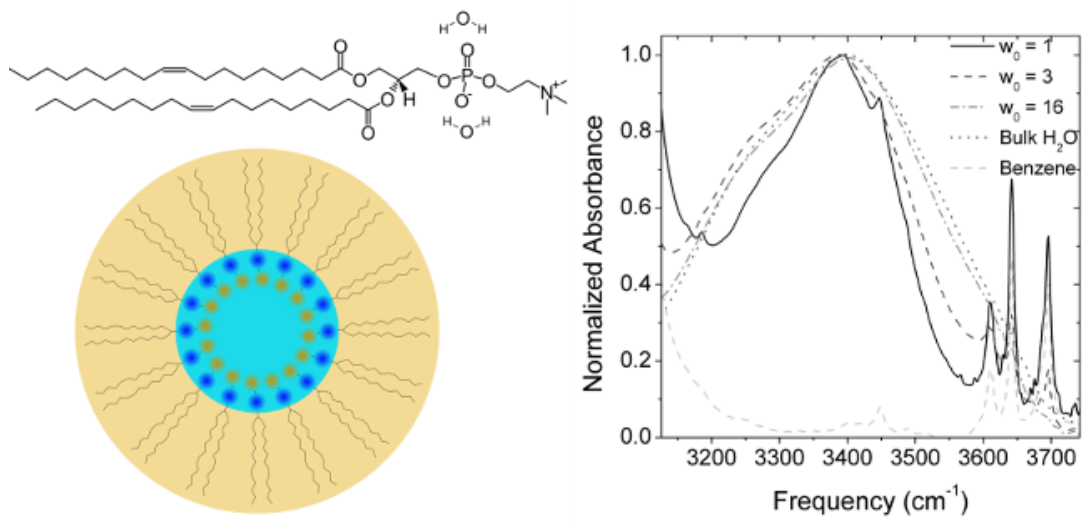

Fig. 1. Structure of DOPC and schematic representation of a DOPC reverse micelle (left); normalized linear absorption spectra of $\mathrm{OH}$ stretching vibrations of water inside DOPC reverse micelles for different hydration levels bulk water and benzene absorptions are shown for comparison (right).

This is an Open Access article distributed under the terms of the Creative Commons Attribution License 2.0, which permits unrestricted use, distribution, and reproduction in any medium, provided the original work is properly cited. 
Ultrafast mid-infrared spectroscopy makes it possible to follow the dynamics of vibrational marker modes of the phospholipid molecules as well as those of the nanoconfined water. A first series of time-resolved measurements involving the antisymmetric phosphate stretching vibration, a sensitive probe for the hydration of the hydrophilic head groups, identified the confined water pool as an efficient heat sink for excess energy deposited in the phosphate groups [1]. Here we present new results providing specific insight into the spectral diffusion and vibrational relaxation dynamics of the $\mathrm{OH}$ stretching and bending vibrations of water inside these reverse micelles [2]. We show that for low hydration samples $\left(w_{0}=1\right)$, the water $\mathrm{OH}$ stretching excitation redistributes via the bending mode of water.

DOPC reverse micelles were prepared by dissolving DOPC (dried over $\mathrm{P}_{2} \mathrm{O}_{5}$ in vacuum) into benzene to yield a $0.25 \mathrm{M}$ solution. We added appropriate amounts of water to obtain reverse micelles with hydration levels in the range of $w_{0}=1$ to 16 . Time-resolved one- and two-color pumpprobe spectra of the $\mathrm{OH}$ stretching and $\mathrm{OH}$ bending vibrations were acquired with pump and probe pulses centered at $3400 \mathrm{~cm}^{-1}$ and between 1600 and $3500 \mathrm{~cm}^{-1}$, (bandwidth $150-200 \mathrm{~cm}^{-1}$ ).

Figure 2 shows results of pump-probe experiments of the $\mathrm{OH}$ stretching mode for $w_{0}=1,3$, and 16. Transient spectra (Figure 2a) at early delay times display an enhanced absorption at low frequencies around $3000 \mathrm{~cm}^{-1}$ and a broad bleaching around $3400 \mathrm{~cm}^{-1}$. While the enhanced absorption decays due to depopulation of the $\mathrm{v}=1$ state, the bleaching undergoes a spectral reshaping and an enhanced absorption builds up at the blue side of the spectrum indicating the formation of a hot ground state. To analyze the spectral reshaping in more detail we calculated the first moment of the bleaching (Figure 2b). For $w_{0}=16$ this analysis is limited to frequencies below $3520 \mathrm{~cm}^{-1}$ to avoid contributions from the pronounced hot ground state absorption. The results of this analysis (Figure $2 \mathrm{~b})$ show an increase of the reshaping time scales from $330 \mathrm{fs}$ for $w_{0}=16$ to $1.4 \mathrm{ps}$ for $w_{0}=1$. For high hydration we attribute the rapid changes to spectral diffusion. The rate of spectral diffusion is slightly slower than for bulk water, where resonant energy transfer between $\mathrm{OH}$ stretching oscillators and fluctuations of the hydrogen bond network play a prominent role [3]. At low
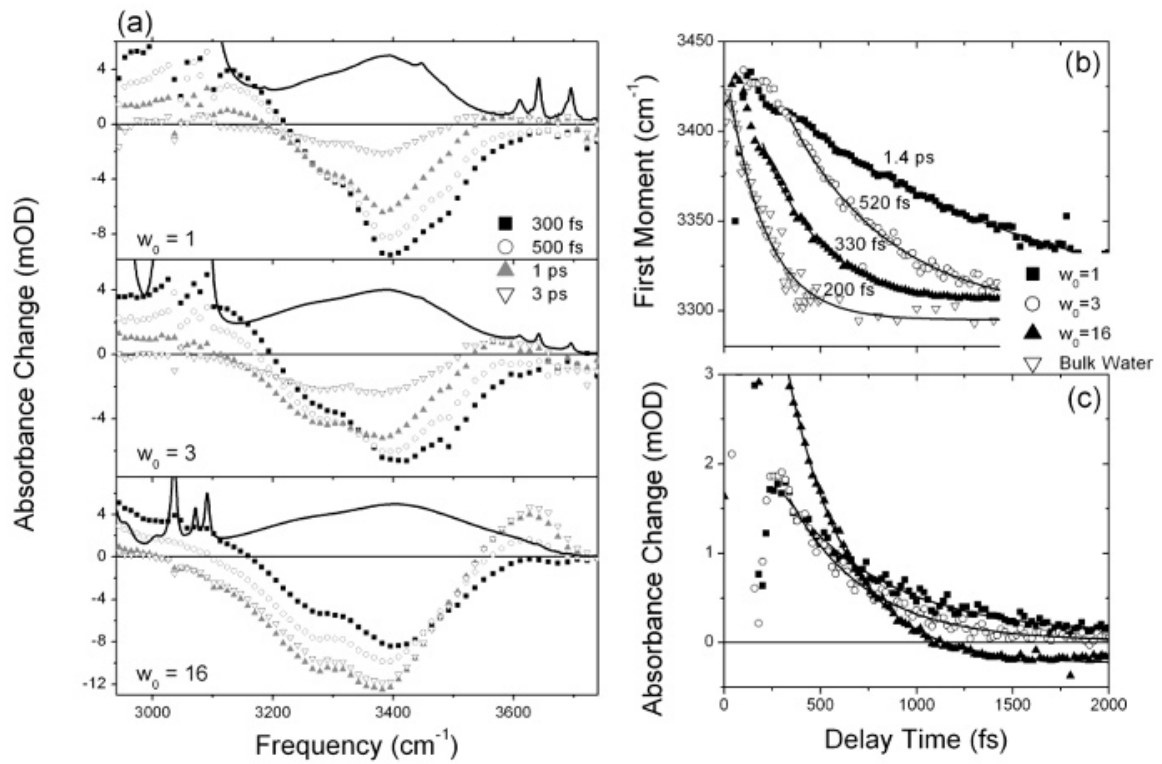

Fig. 2. (a) Transient $\mathrm{OH}$ stretching spectra after excitation with pump pulses centered at $3400 \mathrm{~cm}^{-1}$ for three different hydration levels, solid lines correspond to linear absorption spectra; (b) time-dependent shift of the first moment of the transient spectra with the indicated exponential decay times, values for bulk water [3] are shown for comparison; (c) decay of the excited state absorption at $3004 \mathrm{~cm}^{-1}$ indicating lifetimes of $530 \mathrm{fs}$ $\left(w_{0}=1\right), 400 \mathrm{fs}\left(w_{0}=3\right)$ and $310 \mathrm{fs}\left(w_{0}=16\right)$. 


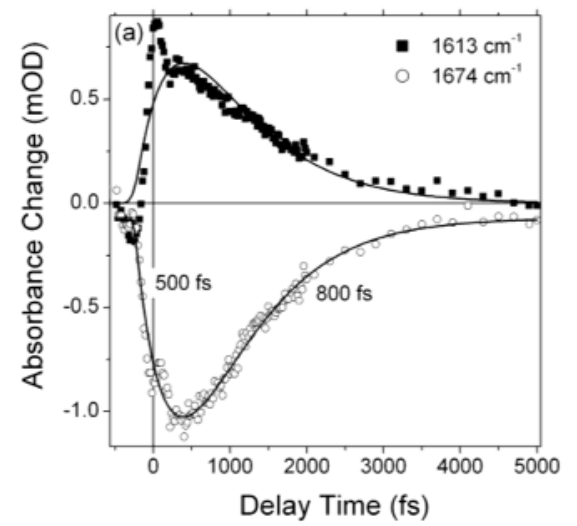

(b)

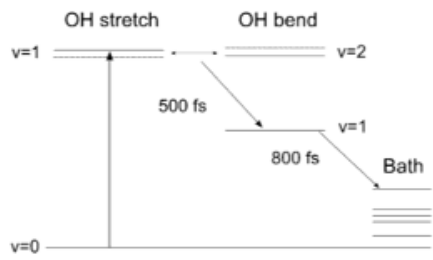

Fig. 3. (a) Transient response of the $\mathrm{OH}$ bending mode after $\mathrm{OH}$ stretching excitation; (b) level scheme with the indicated lifetimes for $\mathrm{OH}$ stretching and bending modes for $w_{0}=1$.

water content $\left(w_{0}=1\right)$ a contribution of the developing hot ground state cannot be excluded. Nevertheless, the $1.4 \mathrm{ps}$ timescale sets a lower limit for the spectral diffusion of single water molecules bound to the phosphate groups as a result of the absence of a fluctuating hydrogen bond network that is present in bulk water.

Figure 2c displays time-resolved pump-probe transients recorded at $3000 \mathrm{~cm}^{-1}$, illustrating the depopulation of the excited $\mathrm{v}=1$ state. It is evident that the lifetime of the $\mathrm{OH}$ stretching mode increases from $\sim 300 \mathrm{fs}$ to $\sim 500 \mathrm{fs}$ when decreasing the hydration level from $w_{0}=16$ to $w_{0}=1$. To understand the increased lifetime, we investigated the energy relaxation pathway with two-color pump-probe measurements monitoring the $\mathrm{OH}$ bending response after $\mathrm{OH}$ stretching excitation. Figure 3(a) shows data for $w_{0}=1$ along with fits that correspond to a rise time of $500 \mathrm{fs}$ and a decay time of $800 \mathrm{fs}$, corresponding to the $500 \mathrm{fs}$ lifetime of the $\mathrm{OH}$ stretching and an $800 \mathrm{fs}$ lifetime of the $\mathrm{OH}$ bending vibration at this hydration level. Our results clearly demonstrate that the $\mathrm{OH}$ bending is involved in the energy relaxation pathway of the $\mathrm{OH}$ stretching mode. This is similar to the case of bulk water - however with different lifetimes [3]. In this scenario, a coupling between the $v=1$ of the $\mathrm{OH}$ stretching and the $\mathrm{v}=2$ of the $\mathrm{OH}$ bending leads to an efficient energy transfer from the former to the latter vibrational mode.

The different $\mathrm{OH}$ stretching lifetimes at different hydration levels $\left(w_{0}\right)$ can be rationalized by considering the slowing down of spectral diffusion with decreasing water content. Because the overtone of the $\mathrm{OH}$ bending mode is located at $3200 \mathrm{~cm}^{-1}$, OH stretching oscillators at the red part of the spectrum can directly relax via the $\mathrm{OH}$ bending mode. Oscillators in the blue part of the spectrum undergo spectral diffusion to bridge the energy gap to the $\mathrm{v}=2 \mathrm{OH}$ bending state. In contrast to bulk water, spectral diffusion occurs on a picosecond timescale for reverse micelles with $w_{0}=1$ resulting in the longer $\mathrm{OH}$ stretch lifetimes. In this way, the absence of the manifold of intermolecular vibrational modes of water at $w_{0}=1$ can explain the energy relaxation of intramolecular vibrational modes. Similarly, the longer $\mathrm{OH}$ bending lifetimes can be explained by the absence of low-frequency librational modes so that the $\mathrm{OH}$ bending mode has to relax via low-frequency modes of the DOPC.

\section{References}

1. N. E. Levinger, R. Costard, E. T. J. Nibbering, T. Elsaesser, J. Phys. Chem. A 115, 11952 (2011)

2. R. Costard, N. E. Levinger, E. T. J. Nibbering, T. Elsaesser, J. Phys. Chem. B 116, 5752 (2012)

3. D. Kraemer, M. L. Cowan, A. Paarmann, N. Huse, E. T. J. Nibbering, T. Elsaesser, R. J.

D.Miller, Proc. Natl. Acad. Sci. USA 105, 437 (2008) 